Open Access

\title{
Design of a Mars atmosphere simulation chamber and testing a Raman Laser Spectrometer (RLS) under conditions pertinent to Mars rover missions
}

\author{
K. Motamedi ${ }^{*}$, AP Colin ${ }^{1}$, JH Hooijschuur ${ }^{1,2}$, O. Postma ${ }^{1}$, R. Lootens ${ }^{3}$, D. Pruijser ${ }^{3}$, R. Stoevelaar ${ }^{3}$, F. Ariese ${ }^{2}$, \\ I B. Hutchinson ${ }^{4}, \mathrm{R}$ Ingley ${ }^{4}$ and GR Davies ${ }^{1}$
}

\author{
* Correspondence: \\ khadijeh.motamedi@falw.vu.nl \\ 'Deep Earth and Planetary Science \\ department, Faculty of Earth and \\ Life Sciences, VU University \\ Amsterdam, De Boelelaan 1085, \\ Amsterdam $1081 \mathrm{HV}$, The \\ Netherlands \\ Full list of author information is \\ available at the end of the article
}

\begin{abstract}
Raman spectrometry is a powerful technique for the rapid identification of most minerals and organic chemicals without sample preparation. In this context, the European Space Agency (ESA) and NASA selected a Raman spectrometer in the payload of the future ExoMars and Mars 2020 missions to identify organic compounds and mineral products indicative of biological activity on Mars. Little is known, however, about the effects of Mars atmospheric conditions on instrument performance and on the Raman spectra. The objective of this study was to i) design and construct a versatile simulation chamber to reproduce the atmospheric conditions expected inside a rover on Mars, ii) to test the performance of a previously designed breadboard miniaturized Raman laser spectrometer (RLS) inside the chamber. The Mars Atmosphere Simulation Chamber (MASC) is a temperature and atmosphere controlled chamber. It includes an innovative heating-cooling system to create homogeneous temperatures inside the chamber that can be varied between $243 \mathrm{~K}$ and $283 \mathrm{~K}$, while the charged coupled device (CCD) of the Raman spectrometer can be independently cooled (e.g., $233 \mathrm{~K}$ ). A vacuum and gas control system permits evacuation of the chamber and the subsequent introduction of any (dried) gas mixture at partial pressures between 1 mbar and several bars. The minimum CCD temperature was found to depend on the surrounding MASC temperature and atmosphere. A vertical shift of 3 pixels on the CCD was observed for the Raman signals upon lowering the temperature from 283 to $253 \mathrm{~K}$. We show that the RLS instrument gives reliable Raman spectra over the tested range of temperatures and from a vacuum of $4 \times 10^{-5} \mathrm{mbar}$ to a $\mathrm{CO}_{2}$ atmosphere at pressure relevant to Mars ( 8 mbar). For example, the Raman spectra of three test minerals, calcite, aragonite and baryte, showed identifiable Raman peaks with Raman shift values within $\pm 1 \mathrm{~cm}^{-1}$ of those reported in previous works under terrestrial conditions. This confirms that a RLS instrument is useful for the identification of minerals during future missions to Mars; once a necessary detector recalibration was carried out, the system performed well under 8 mbar pressure and $243-283 \mathrm{~K}$. The MASC was found to be a versatile instrument; it can provide important information on instrument performance under Martian conditions and other temperatures and atmospheric conditions can also be simulated.
\end{abstract}

Keywords: Mars simulation chamber; Cooling-vacuum system; Martian atmosphere; Raman laser spectrometer

\section{严 Springer}

(c) 2015 Motamedi et al. licensee Springer on behalf of EPJ. This is an Open Access article distributed under the terms of the Creative Commons Attribution License (http://creativecommons.org/licenses/by/4.0), which permits unrestricted use, distribution, and reproduction in any medium, provided the original work is properly credited. 


\section{Introduction}

Fundamental questions remain about the geological history of the planet Mars [1-3]. One key question is whether free water has been persistent on the Mars surface to support life. The presence of unaltered recent volcanic rocks and minerals (e.g., olivine) suggests that the surface is currently dry $[4,5]$. Previous studies, however, have shown that the planet experienced major climate changes and that the earlier conditions were warmer and wetter [6]. For example, the weathering and erosion products are evidence of the presence of water in the early geological history of Mars, during the Noachian era [5-7]. The geographic extent of minerals such as hydrated, evaporite or sedimentary minerals (e.g., hydrated sulphates, carbonates, phyllosilicates) is still being determined and how long this wet period lasted is still matter of debate [6-8]. Therefore, planetary scientists are interested in the accurate in situ determination of the surface mineralogy and mineral composition on Mars by advanced techniques and instruments on board Mars landers and rovers [9-14]. The instruments are usually miniaturized versions of those routinely used on Earth. Little is known, however, about the effects of different atmospheric conditions on the performance of such instruments and on the response of the minerals to be analysed.

Kolesov and Geiger reported that the temperature may affect the structure of minerals and the Raman peak shape and intensity [15]. In the case of techniques that use plasmas, such as Laser-induced breakdown spectroscopy (LIBS), the characteristics of the plasma and its expansion depends on the ambient pressure [16] and thermal distortions of the components of the instrument itself can affect its calibration or performance. Thus it is essential to simulate conditions similar to those expected during the space missions, in order to predict and take into account the accuracy and precision of instrumental data, or if necessary adapt the design.

Worldwide there are several atmospheric simulation chambers that are capable of reproducing Martian conditions to some extent [17-19]. In the context of testing instrument, however, many are too small and do not allow housing an instrument and samples simultaneously. The instrument and the samples to be analysed are usually separated physically by viewports/windows and the experiments are not performed under fully realistic conditions. Therefore, we designed and built a versatile Mars Atmosphere Simulation Chamber (MASC) with a large volume to house both the instruments and the samples, and to simulate the conditions inside a rover during the next space missions to Mars.

In this paper, we first describe the design of the new MASC chamber and the protocol for experiments under the conditions expected inside the rover during the ExoMars mission. Finally, we test a prototype Raman Laser Spectrometer (RLS) instrument previously designed for the ExoMars mission by the Dutch Organization for Applied Research (TNO) on a set of three test minerals.

\section{Design requirements and technical description of the Mars Atmosphere Simulation Chamber (MASC) and Raman Laser Spectrometer (RLS)}

The MASC is a cylindrical stainless steel chamber, volume $0.261 \mathrm{~m}^{3}(100 \mathrm{~cm}$ length and a diameter of $57.6 \mathrm{~cm}$ ), with doors at each end to ensure a high versatility. Previous work related to ExoMars Raman studies by Sobron and Wang [20] estimated that the rover operation temperature range would be between $243 \mathrm{~K}$ and $283 \mathrm{~K}$ during Raman measurements and we await final operating conditions to be released by ESA. The major 
requirement in the design of the chamber was to provide stable temperatures and pressures (6-10 mbar of pure $\mathrm{CO}_{2}$ atmosphere and a temperature range between $243 \mathrm{~K}$ and $283 \mathrm{~K}$ ) that could be varied in line with experimental requirements. In addition, rapid changes in temperature would subject the optics of space instruments to thermal stresses. Therefore, it was required to control the rate of heating or cooling of different parts of the MASC. To fully assess the effect of low pressure on the instrument performance, it was desirable to design a chamber with the flexibility to produce stable pressures from a vacuum $(\sim 4 \times 10$ ${ }^{-5}$ mbar) to a pressure of a 6-10 mbar of $\mathrm{CO}_{2}$ as expected on Mars. Moreover, the water content inside the MASC had to be kept low, for example on Mars the water content is $0.03 \%[21]$.

The chamber contains a variety of electrical/optical feed-throughs, viewports and vacuum measurement gauges, as well as other instruments. A sample holder was fitted with 3D stages so that different samples could be analysed without the need for opening the chamber in between sample analyses. As the majority of planetary instruments will include CCDs as detectors (e.g., for ExoMars Cameras, X-ray Diffraction (XRD), X-ray fluorescence (XRF) and Raman laser spectrometer (RLS instrument), the MASC was fitted with a separate cooling circuit for the CCD of the space instrument.

The main doors provide support for mounting plates. For example, in the context of testing the RLS instrument (see below) one mounting plate supports the RLS spectrometer and another carries the optical head and sample holder with a 3D stage, see Fig. 1.

\section{Temperature control}

The MASC was insulated from the ambient laboratory conditions by a double wall, with an interstitial space of $60 \mathrm{~mm}$. This enabled us to reach a $10^{-6}$ mbar vacuum over the full length of the chamber, after several cycles of pumping and outgassing at $393 \mathrm{~K}$.

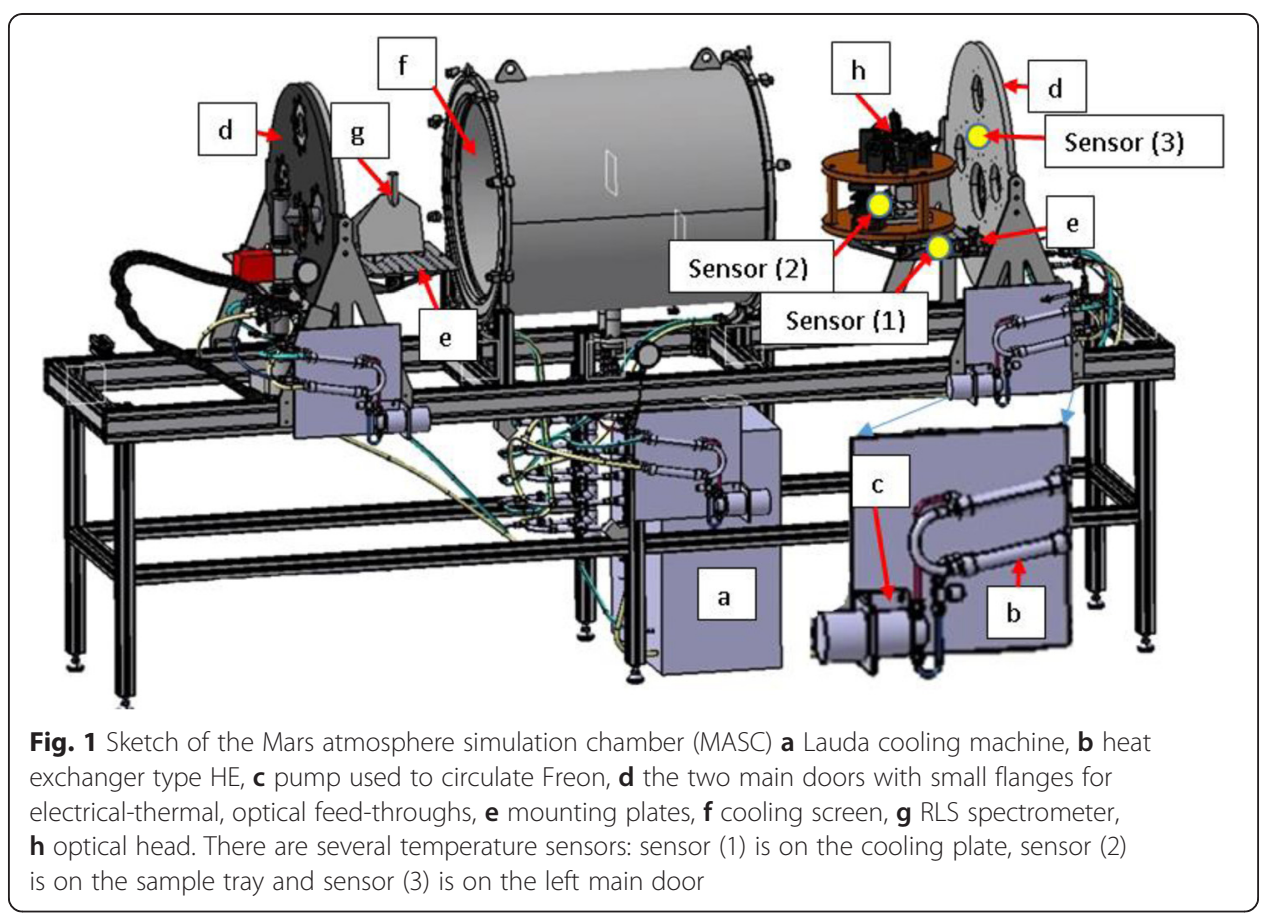


In addition, the outer wall and the two main doors of the MASC were covered with a flexible $20 \mathrm{~mm}$ thick insulation layer of Thermoplastic elastomeric foam (TPE).

To cool/heat the main doors, a Lauda XT-150 cooling machine, with silicone oil as the cooling medium, was used. The main doors were produced from a single piece of steel such that the silicone oil cannot leak inside the chamber and contaminate the instruments. The Lauda was considered a simple, safe and reliable machine with an accuracy of $\pm 0.05 \mathrm{~K}$. The power outputs of the Lauda at 293 and $233 \mathrm{~K}$ were 1.50 and $0.06 \mathrm{~kW}$ respectively, which was sufficient for the heating/cooling of the MASC when operated between 243 and $283 \mathrm{~K}$.

The inside of the chamber, i.e. the cooling screen and mounting plates, upon which experiments were performed or instruments tested, needs to be temperature controlled. Due to their design structure and position inside the MASC there would be a risk that these parts could leak and contaminate the chamber interior. Therefore, we used a non-corrosive cooling medium in a secondary closed system in thermal contact through heat exchanges with the primary silicone cooling circuit. Freon $\left(\mathrm{C}_{2} \mathrm{Cl}_{3} \mathrm{~F}_{3}\right)$ was selected as a safe cooling medium (nontoxic and non-flammable) and was passed through cooling pipes in the mounting plates and the cooling screen in a liquid state (at $\sim 6$ bars at room temperature).

The cooling screen was made of two stainless steel plates that were partially welded together in such a way that it formed inter-connected channels. In order to produce a pillow shape, the welded plates were pressurised to $\sim 60$ bars. This caused the plates to inflate like a pillow. As shown in Fig. 2, there are six welds (concentric circles) in the design of the cooling plate to create the cylindrical tube. The two mounting plates were cooled via cooling pipes located below the plates. To ensure flexibility in temperature control within the chamber, three secondary circuits where manufactured, each with a dedicated pump and heat exchanger. Pumps were designed (Micro Pump Company) for Freon use in the temperature ranges from 227 to $450 \mathrm{~K}$. Two pumps with flow capacities of $380 \mathrm{ml} / \mathrm{min}$ circulated Freon from the heat exchangers to the mounting plates. As the cooling screen had the largest volume and was mounted higher than mounting plates, a pump with a higher capacity $(930 \mathrm{ml} / \mathrm{min})$ was needed to pump Freon to the cooling screen. Heat was transferred from the silicone oil to the Freon by a counter flow principle, via a heat-exchanger (type HE 0.5 from Danffos, $178 \mathrm{~mm}$ length and weighs $0.3 \mathrm{~kg}$ ). The cooling capacity of the HE 0.5 type was $\sim 0.33$ and $4 \mathrm{~kW}$ at 233 and $293 \mathrm{~K}$ respectively [22].

The cooling system was filled to $90 \%$ of the volume to allow expansion of the Freon whenever the temperature was raised. Currently the temperature inside the chamber can be varied between 243 and $283 \mathrm{~K}$, but the stainless steel structure of the chamber allows the temperature to be varied over a larger range of temperatures if required.

\section{Protection of the electrical/ optical feed-through on the doors}

Initial cooling experiments with the MASC produced condensation on the surface of the small flanges/feed-throughs of each door at a temperature near or below $290 \mathrm{~K}$. This potentially could lead to problems for electronic and optical components. Therefore, flanges/feed-throughs were maintained above $293 \mathrm{~K}$ by the use of power resistors. These have a low resistance (1-2 Ohms) and can handle up to 30 Watts. A Call9300 (CAL 


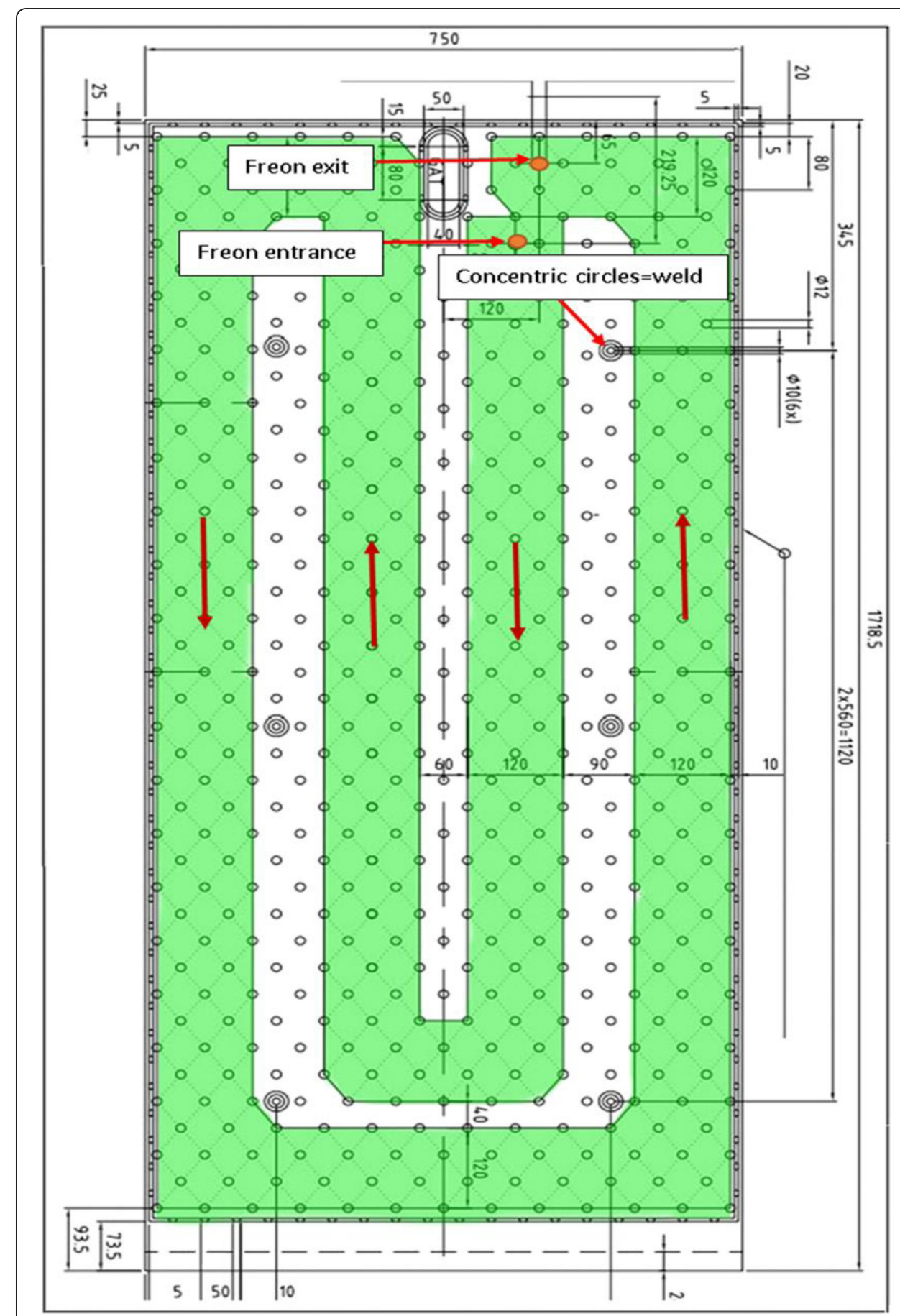

Fig. 2 Design and dimensions $(\mathrm{mm})$ of the cooling plate; Freon enters from the red entrance and is passed along the green path inside the cooling plate. The circles indicate the connection of cooling plates but allow Freon to flow between the welds (area in green). There are six welds (concentric circles) plus one large oval weld in the cooling plate that connects the plates and prevents Freon circulation which is shown in the white area 
Controls, UK) temperature controller was used to set the temperature. Therefore, each door could be set to a desired temperature (see Fig. 3).

\section{Atmosphere control inside the MASC}

Simulated Martian conditions were achieved by using an atmosphere gas control system. For simplicity, initial testing was conducted with $100 \% \mathrm{CO}_{2}$, which represents a reliable proxy for the Martian atmosphere. The chamber, however, has the flexibility to simulate different gas compositions and pressures. Also, by using copper O-rings and a larger turbo molecular pump, it will be possible to obtain a lower vacuum for testing instrument under lunar conditions.

Martian pressure conditions were simulated inside the MASC by first evacuating the chamber and then flushing with $\mathrm{CO}_{2}$. To maintain stable Martian conditions, the MASC needs to be leak tight. EPDM O-rings (ethylene-propylene-diene rubber, hydrogen peroxide cured) were chosen for the large entrance doors because of their resistance to low temperature (down to $233 \mathrm{~K}$ ) in combination with their compatibility to clean applications in the pharmaceutical/food industry.

The fact that the Martian atmosphere only contains $0.03 \%$ water also had to be considered. The large surface area of the chamber's wall, main doors and multiple electrical/ optical feed-throughs could lead to extensive outgassing. The vacuum system comprises an oil free dry-compression diaphragm pump (Pfeiffer PKT01300) that was powered by a turbo molecular pump (Pieffer TMH 071P) to reach an initial vacuum of $\sim 10^{-5}$ mbar. Even when the chamber contained a spectrometer, an optical head, samples and all optical fibres and electrical cables, the pump down time was rapid and $\mathrm{H}_{2} \mathrm{O}$ was quickly and effectively removed from the system without the need for an additional cold finger (the pressure dropped below $4 \times 10^{-5}$ mbar in 2 hours). In addition, a parallel arrangement of

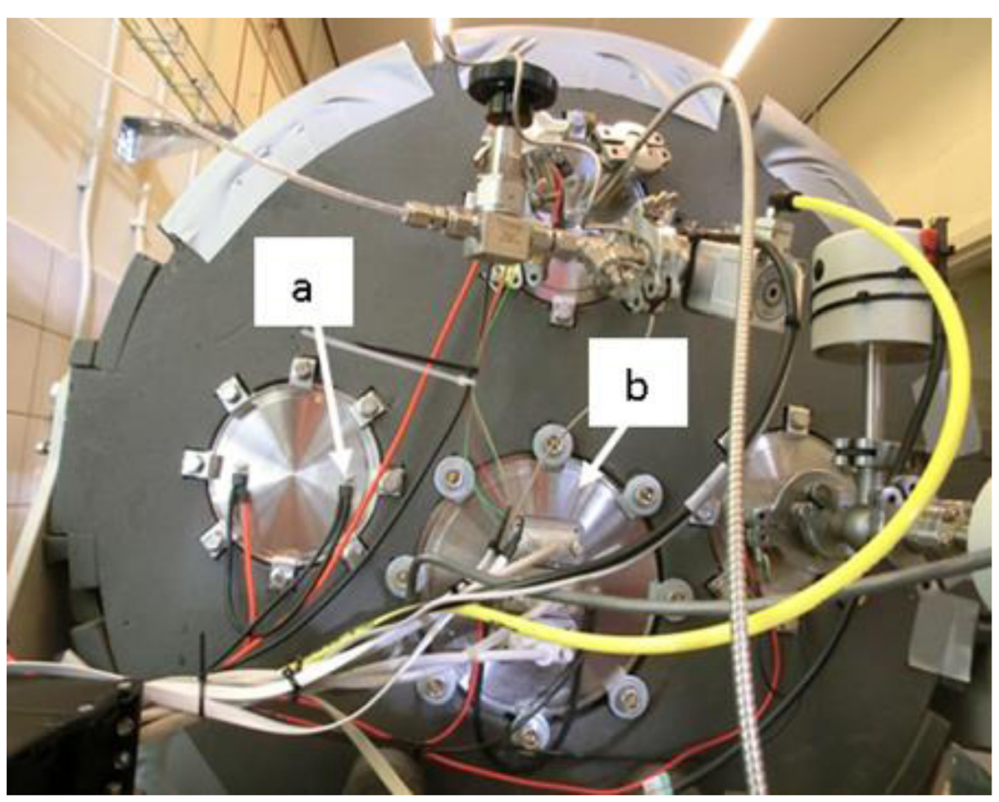

Fig. 3 View of one of the MASC doors with a) local heater and b) a flange fitted with optical or electrical feed-throughs 
Nafion tubes cleans the $\mathrm{CO}_{2}$ gas of water. This method of water removal uses a dry helium flow around the permeable Nafion tube to flush the water across the membrane wall [23]. A particle filter of $0.5 \mathrm{~mm}$ mesh was mounted on the $\mathrm{CO}_{2}$ feeding line to prevent deposition of solid particles in the tank.

\section{Cooling the CCD of the RLS}

An additional cooling system was implemented to minimize thermal noise/ dark noise of the CCD in the RLS instrument. In our cooling design of the CCD, a Peltier cooler was mounted on the CCD and connected via a braided copper thermal strap ( $\sim 25 \mathrm{~mm}$ wide and $5 \mathrm{~mm}$ thick) to a copper cooling block. Inside the MASC, this copper cooling block was mounted to a small flange in the chamber door. From the outside of the MASC, the copper cooling block was connected to an Etscheid water cooler with the cooling capacity of $750 \mathrm{kcal} / \mathrm{h}$ at $288 \mathrm{~K}$. This cooler was located directly under the chamber to avoid unnecessary heat exchange.

\section{Design of a sample holder with 3D linear stages}

Efficient use of the chamber required that multiple samples could be analysed sequentially. Three linear positioning stages (manufactured by the Physik Instrumente Company, Germany) were combined to form a 3D linear stage. These three linear positioning stages can carry up to $20 \mathrm{~kg}$, push/pull up to $50 \mathrm{~N}$ and have an accuracy of about $\pm 0.1 \mathrm{~mm}$. A sample holder was mechanically connected to the upper linear stage. The sample tray was $83 \mathrm{~mm}$ in length and $35 \mathrm{~mm}$ wide and could hold ten samples. Samples were solid pieces of mineral mounted in epoxy (cylinders with diameters of $10 \mathrm{~mm}$ ) and $3 \mathrm{~mm}$ apart to allow potential use of laser ablation techniques.

\section{Design of the CCD detector}

The RLS instrument used for this study was an elegant breadboard for the ExoMars mission, originally designed as a combined Raman and Laser Induced Breakdown Spectroscopy (LIBS) instrument [24]. In order to decrease the weight and power usage of the payload aboard the ExoMars mission, the LIBS capability had in the meantime been removed from the flight mode instrument. Therefore, the effect of Mars atmospheric conditions on the LIBS performance of the instrument is not discussed in this article. The Raman modality uses a $659 \mathrm{~nm}$ laser with a maximum output of $20 \mathrm{~mW}$. The RLS focal plane assembly incorporates a charge coupled device (CCD), a thermal/mechanical interface (cold finger) and proximity electronics. The proximity electronics include CCD bias voltage filters and a pre-amplifier circuit for amplifying the analogue video output signal produced by the on-chip amplifier. The thermal design of the spectrometer allows the $\mathrm{CCD}$ to be operated between $-40{ }^{\circ} \mathrm{C}$ and room temperature, with the $\mathrm{CCD}$ thermally isolated from the rest of the spectrometer including the proximity electronics. The spectrometer was designed to project multiple grating orders at different heights of the CCD. This way the instrument could be used over a broad wavelength range (for LIBS) and still offer a high spectral resolution (for Raman and LIBS). The CCD was a backilluminated e2v Technologies CCD42-40 with an imaging area comprising $2048 \times 2048$, $13.5 \mu \mathrm{m}$ pixels. The device is Ultraviolet (UV)-enhanced, with a quantum efficiency of at least $50 \%$ between 240 and $840 \mathrm{~nm}$ and imaging pixels with a full-well capacity in excess 
of $10^{5}$ electrons. The device is a full-frame CCD but is usually operated in pseudo frame transfer mode in order to reduce smearing effects at short integration times (which can result in cross contamination of the spectral orders). The CCD is bonded to a ceramic Dual-In-Line (DIL) package but is flight representative with respect to its functional and thermal performance (including the mechanical interface to the rest of the spectrometer). The analogue video output from the focal plane assembly is digitised using a correlated double-sampling circuit and low-noise analogue-to-digital converter. A programmable digital signal processor (DSP) enables flexible detector operating modes, including synchronised CCD readout and laser emission.

\section{Results}

\section{Temperature control inside the MASC}

A representative low temperature experiment under Martian conditions inside MASC consists of five stages: (1) loading the sample tray, (2) evacuating the MASC to remove all water ( $4 \times 10^{-5}$ mbar), (3) pumping 8 mbar of $\mathrm{CO}_{2}$ into the MASC, (4) setting the Lauda cooling machine to a specific temperature (5) recording the temperature data.

Dallas 1-wire temperature sensors (type DS18B20) were used to monitor the temperature behaviour as a function of time within the chamber. A total of twenty two sensors are used to measure the ambient $\mathrm{CO}_{2}$ temperature inside the chamber. A small electronic interface that connects the sensors to a computer, allows monitoring the temperature throughout the chamber. The temperature sensors were mounted to the MASC components using vacuum compatible glue. Sensor (1) on the left cooling plate, sensor (2) on the sample tray and sensor (3) on the left main chamber door are also shown in Fig. 1. Sensor 4 was glued on the middle of the cooling screen. An example of temperature change with time on the left cooling plate and the left side of the chamber wall is present in Fig. 4 and shows that temperatures were stable after 15 hours.

The sensors on the left cooling plate front and the middle of the chamber were directly cooled by the Freon passing through the cooling pipe and cooling screen. Consequently, these sensors recorded the lowest temperature of $\sim 244$ and $248 \mathrm{~K}$ respectively. In contrast, due to the open structure of the 3D linear stages, there was relatively poor thermal conductivity between the cooling plate and the sample tray. Thermal straps were included in the design but better thermal conduction is needed to reduce the sample tray below $\sim 250 \mathrm{~K}$. The temperature on the left door only reached $\sim 285 \mathrm{~K}$, because local heaters maintain the electrical feed-throughs at $293 \mathrm{~K}$. Therefore an aluminium cover was designed to insulate the inside of the MASC from thermal radiation of the doors.

\section{Cooling of the CCD}

Experiments were performed to assess the cooling system capabilities of the CCD detector inside the MASC. MASC was operated under a vacuum of $4 \times 10^{-5}$ mbar or with 8 mbar of $\mathrm{CO}_{2}$, at temperatures of 281, 265 and $238 \mathrm{~K}$. The minimum CCD temperatures achieved under vacuum were 241, 237 and $232 \mathrm{~K}$ respectively. In contrast, in the presence of $\mathrm{CO}_{2}$, the minimum $\mathrm{CCD}$ temperatures were higher: 256, 248 and $239 \mathrm{~K}$. The temperature response of the CCD over time is presented in Fig. 5. Irrespective of the MASC operating temperature, the CCD always reached a temperature below $243 \mathrm{~K}$ under 


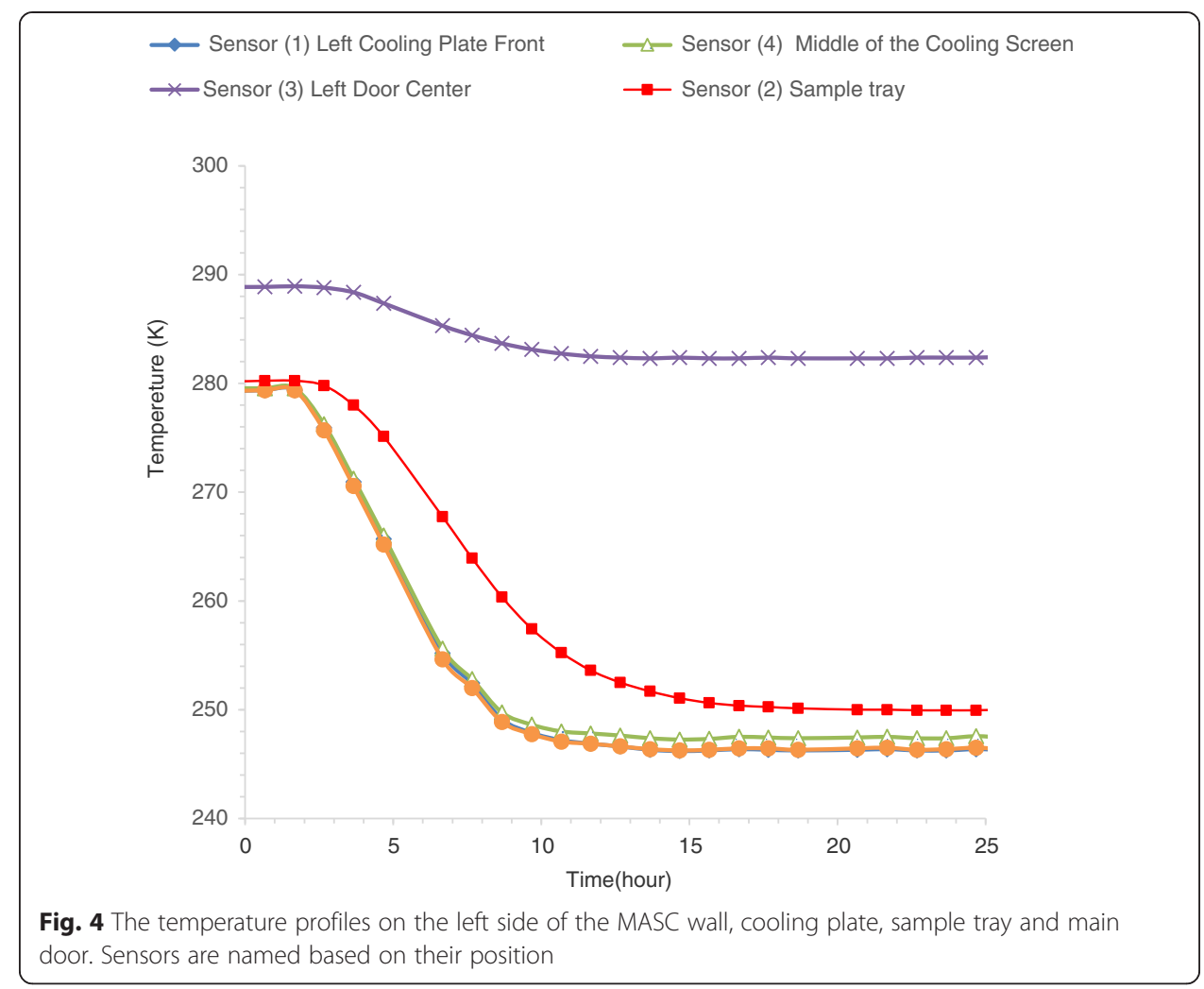

vacuum conditions. In a $\mathrm{CO}_{2}$ atmosphere, when the MASC operational temperature was at 281 and $265 \mathrm{~K}$, the $\mathrm{CCD}$ reached temperatures of 256 and $248 \mathrm{~K}$ respectively. At a MASC temperature of $238 \mathrm{~K}$ the Peltier system did not provide additional cooling. These data indicate that further work is required to improve the cooling design of the CCD, particularly in the presence of an atmosphere. Redesign is underway to improve thermal contact between interfaces and greater insulation of the $\mathrm{Cu}$ block and braided strap.

\section{Calibration testing of the CCD}

Data acquisition used a single $40 \mathrm{~s}$ integration. With the $659 \mathrm{~nm}$ laser, the most relevant Raman scattering modes can be detected on the 3rd diffraction order between 660 and
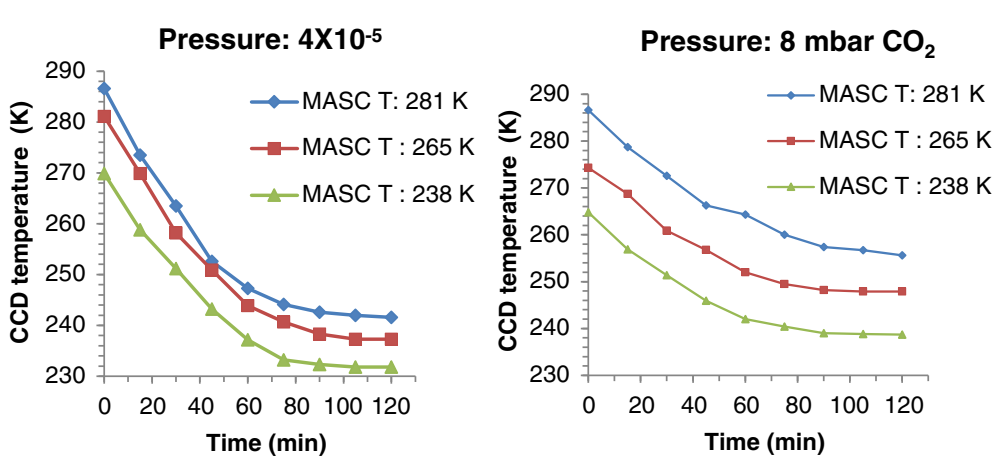

Fig. 5 Temperature response of the CCD over time under vacuum condition $\left(4 \times 10^{-5} \mathrm{mbar}\right.$, left panel) and $8 \mathrm{mbar} \mathrm{CO}_{2}$ pressure (right panel), when the temperature of the MASC was 281,265 and $238 \mathrm{~K}$ 
$840 \mathrm{~nm}$. The spectral resolution was $\sim 0.2 \mathrm{~nm}\left(\sim 4 \mathrm{~cm}^{-1}\right)$, i.e. doublet peaks separated by less than $4 \mathrm{~cm}^{-1}$ cannot be fully resolved. In an independent set of experiments, the same resolution of the spectrometer was determined under ambient conditions based on the peak width measured for the sharp line of a mercury calibration lamp: $0.2 \mathrm{~nm}$ FWHM [24-26]. The accuracy of Raman shift values, however, was $\sim 0.4 \mathrm{~cm}^{-1}$. For Mars missions this accuracy would be sufficient to distinguish between minerals such as calcite and aragonite (a polymorph of calcite, both having the chemical composition $\mathrm{CaCO}_{3}$ but with a different molecular structure). These two minerals have lattice vibration modes (collective vibrations within the crystals) at $\sim 283$ and $274.5 \mathrm{~cm}^{-1}$ respectively, see below.

The calibration of the CCD was tested as a function of the MASC temperature, focussing on the 3rd order where the Raman peaks are located. First, to localise the position of the 3rd order line on the camera, the CCD was illuminated with natural white light; then to precisely calibrate the $\mathrm{CCD}$, the camera was illuminated with neon and argon lamps that produce narrow, intense peaks with known wavelengths between 660 and $840 \mathrm{~nm}$. The calibration results demonstrate that the vertical position of the 3rd diffraction order on the CCD was influenced by temperature and shifted by 3 pixels toward the top of the camera ( $\mathrm{Y}$ direction) when we decreased the temperature from $283 \mathrm{~K}$ to $253 \mathrm{~K}$ (see Fig. 6). In contrast, there was no significant shift in the $\mathrm{X}$ direction (less than a pixel). Indeed, we performed a calibration of the wavelength as a function of the pixel column number (X direction) using $\mathrm{Ar}$ and $\mathrm{Ne}$ lamps at $283 \mathrm{~K}$, then we decreased the temperature of the MASC to $253 \mathrm{~K}$ and illuminated again the CCD with Ar and Ne lamps: when using the calibration performed at $283 \mathrm{~K}$ for this lower temperature, the measured wavelengths are shifted less than $0.07 \mathrm{~nm}$ compared to the real wavelength values. This difference is within our Raman resolution capabilities and thus was not taken into account for our mineral analyses, but suggests that if the CCD is operated at even lower temperatures a specific calibration check might be required. As such, the instrument is useful to detect minerals under Martian conditions but further work is needed to improve the RLS spectrometer design to minimize offset of the spectrum in the $\mathrm{Y}$ direction. More analysis is required to see if thermal expansion of optical components or the mounting assembly caused the vertical offset. Currently, different readout protocols are required to optimize the light collection at a specific temperature.

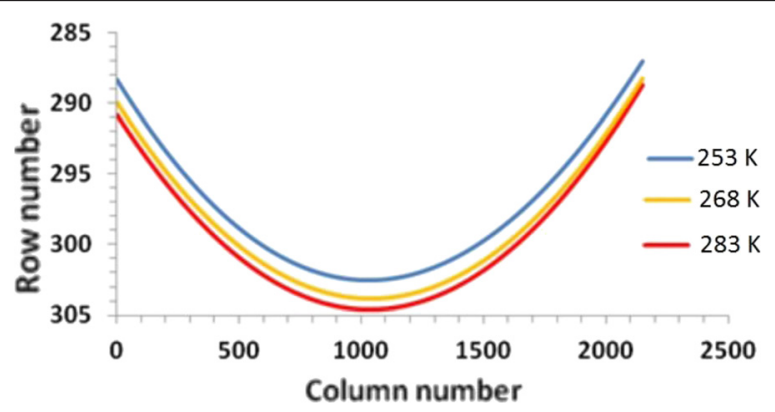

Fig. 6 Temperature-induced shift of the observed white light emission in 3rd order on the CCD at 283, 278 and $253 \mathrm{~K}$. The $X$ axis shows the column number of CCD pixels; the $Y$ axis shows the row number of a part of the CCD pixels, vertically expanded for clarity. The pixel size is $13.5 \mu \mathrm{m}$ square 


\section{Raman spectroscopy}

To assess the capability of the RLS instrument under Martian environmental conditions, we studied the Raman spectra of calcite, aragonite and baryte inside the MASC at different temperatures and pressures. The choice of those three minerals relates to ExoMars' priority objective of searching for signs of water in the shallow subsurface. Indeed, calcite can form in sedimentary, igneous and metamorphic rocks. Aragonite is formed by biological and physical processes, including precipitation from marine and freshwater environments [27] and baryte can also be an indicator of hydrothermal circulation $[28,29]$.

Raman spectra of selected minerals were processed using the Origin program and Lorentzian peak fitting was used to provide a more accurate estimation of the height, position and width of the Raman peaks of the selected minerals for this study. To estimate the repeatability of this fitting routine, we acquired the Raman spectrum of a calcite sample ten times and then fitted the $1087 \mathrm{~cm}^{-1}$ peak for the ten individual spectra. The measurements show that this routine can fit the data and find that the value of a Raman peak shift varies within a standard deviation of $0.5 \mathrm{~cm}^{-1}$, which in the wavelength range in question corresponds to $\sim 0.02 \mathrm{~nm}$.

The spectra of selected minerals are shown in Figs. 7, 8, 9. This study focuses on instrument capabilities by examining minerals under Mars simulation conditions. Such a study is important to further understand how the instrument's performance will change at the low temperatures pertinent to Mars and also if the structure of minerals varies with atmospheric conditions, for example possible changes in the length of atomic bonds [30-33].

In Fig. 7b, the RLS Raman spectrum of calcite (obtained under vacuum condition at $283 \mathrm{~K}$ ) is characterized by the most intense $v_{1}$ symmetric stretching of the $\mathrm{CO}_{3}$ group at $1087.5 \mathrm{~cm}^{-1}$, the $v_{4}$ symmetric bending mode at $713.4 \mathrm{~cm}^{-1}$, and one lattice vibration mode at $282.9 \mathrm{~cm}^{-1}$. These peaks are within $\sim 1 \mathrm{~cm}^{-1}$ of the data reported in previous literature studies [34]. The possible $2 v_{2}$ and $v_{3}$ peaks were not detected with the RLS instrument. These weak peaks appear to have been obscured within the background noise. The Raman spectra of calcite under vacuum condition at $253 \mathrm{~K}$ (see Fig. 7a) or at $8 \mathrm{mbar} \mathrm{CO}_{2}$ (see Fig. 7c) has peaks with Raman shift within $\sim 0.5 \mathrm{~cm}^{-1}$ of those obtained under vacuum condition at $283 \mathrm{~K}$, a scatter which is comparable to the standard error obtained for repeated measurements without changing the temperature and pressure conditions.

Similarly, Figs. 8, 9 also demonstrate that peak positions resolved in the Raman spectra of aragonite and baryte obtained under Martian conditions were within $\sim 0.5 \mathrm{~cm}^{-1}$ of those obtained under vacuum condition at $283 \mathrm{~K}$. These results again strongly suggests that there were no significant differences in the Raman spectra related to Martian conditions (low temperature or $\mathrm{CO}_{2}$ atmosphere). The aragonite spectrum shows: the very strong $v_{1}$ Raman peak at $\sim 1086 \mathrm{~cm}^{-1}$, the $v_{4}$ vibration mode at $\sim 704 \mathrm{~cm}^{-1}$ and a weak lattice vibration modes at $212 \mathrm{~cm}^{-1}$ [35]. In the baryte spectrum, the most intense peak, the $v_{1}$ symmetric stretching vibration mode of $\mathrm{SO}_{4}$ tetrahedra, occurs at $\sim 989 \mathrm{~cm}^{-1}$. The peak at $461 \mathrm{~cm}^{-1}$ is attributed to the $v_{2}$ bending vibration mode. The $v_{3}$ asymmetric stretching vibration and $v_{4}$ asymmetric bending vibration modes are observed at $\sim 1144 \mathrm{~cm}^{-1}$ and $619 \mathrm{~cm}^{-1}$ [36]. Also for these two minerals the spectra fully agree with reference spectra from the literature. 


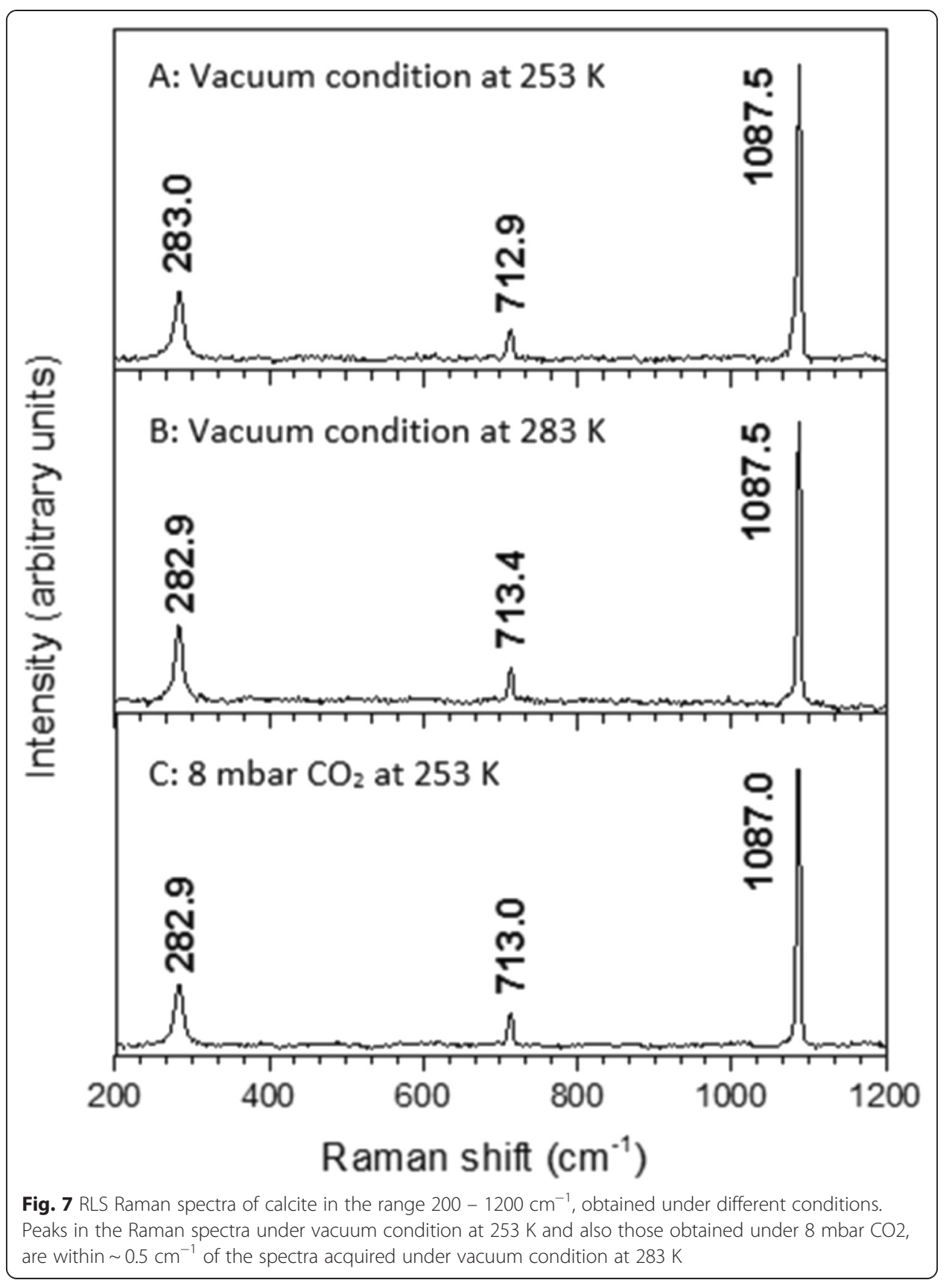

\section{Conclusion}

The recently developed MASC at VU University in Amsterdam reached most of its initial design goals as it is able to simulate Martian environmental conditions. The designed operational temperature range inside the MASC was 283 to $243 \mathrm{~K}$ and the pressure range was 8 to $10^{-5}$ mbar. The unique design of the MASC, having a large volume $\left(0.261 \mathrm{~m}^{3}\right)$ and a separate cooling circuit with a stable low temperature, allowed detailed in situ characterisation of the RLS instrument and samples under Martian conditions. The MASC is not restricted to simulate Martian conditions but can also be used for a whole range of other experiments where a controlled atmospheric composition and temperature is required. 


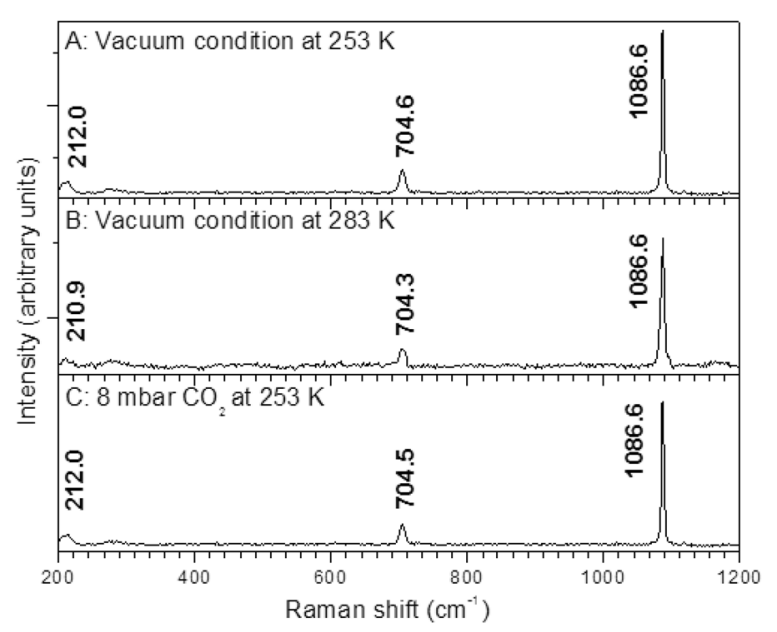

Fig. 8 RLS Raman spectra of aragonite in the range $200-1200 \mathrm{~cm}^{-1}$, obtained under different conditions

The tested RLS spectrograph showed a vertical offset of about 3 pixels when lowering the temperature from 283 to $253 \mathrm{~K}$. This was corrected for by adapting the read-out protocols, and this problem is not likely to occur in a more conventional Raman spectrometer in first order. Nevertheless, for any instrument to be employed in space it is crucial to check the repeatability and calibration under the expected environmental conditions. No significant horizontal shift on the CCD was observed when lowering the temperature.

The data indicated the accurate performance and calibration of the RLS instrument. Raman spectra obtained under Martian conditions (8 mbar $\mathrm{CO}_{2}$ pressure and $253 \mathrm{~K}$ ) were indistinguishable from those obtained under vacuum conditions at a temperature of $253 \mathrm{~K}$ and also indistinguishable from those measured at $283 \mathrm{~K}$. There was also no significant difference in peak positions of the Raman spectra of calcite, aragonite and baryte under Martian conditions in comparison with literature reference spectra recorded under Earth conditions.

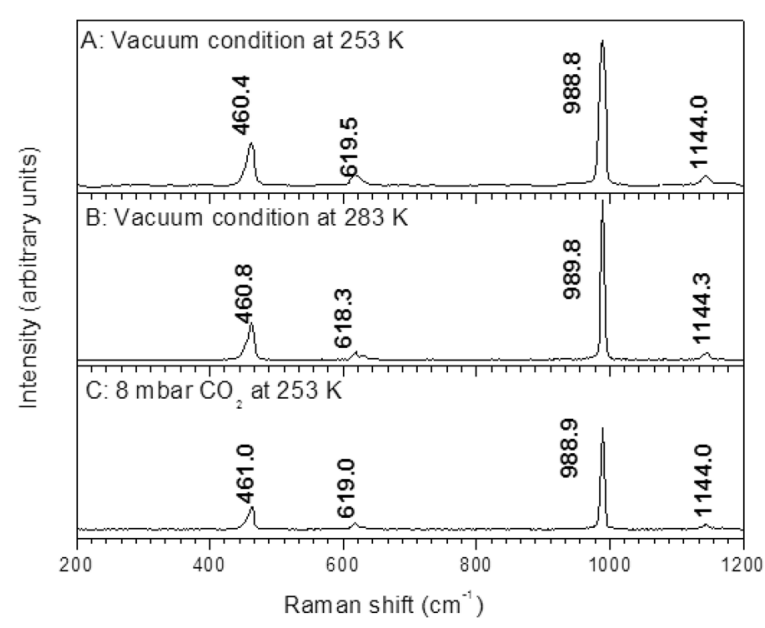

Fig. 9 RLS Raman spectra of baryte in the range $200-1200 \mathrm{~cm}^{-1}$, obtained under different conditions 


\section{Competing interests}

The authors declare that they have no competing interests.

\section{Authors' contributions}

KM carried out the experiments, collected and analysed the data, and wrote the manuscript. KM, OP, RL, DP and RS built up the chamber and designed the experiments. IB and RI made the Matlab script to run the RLS, and helped with installing the instrument inside the MASC. APC made the Matlab script to calibrate the RLS CCD and to extract the Raman spectra from the CCD images. APC and JHH commented the manuscript. FA and GRD improved the manuscript by discussing the results and the draft structure. All authors read and approved the final manuscript.

\section{Acknowledgments}

This work was funded by the Netherlands Organization for Science Research (NWO) (No ALW-GO-PL/07-09 and ALW-GO-PL/14). The funding agency played no role in the data interpretation or the writing of the manuscript.

\section{Author details}

${ }^{1}$ Deep Earth and Planetary Science department, Faculty of Earth and Life Sciences, VU University Amsterdam, De Boelelaan 1085, Amsterdam $1081 \mathrm{HV}$, The Netherlands. 'LaserLaB, Faculty of Sciences, VU University Amsterdam, De Boelelaan 1083, Amsterdam 1081 HV, The Netherlands. ${ }^{3}$ Electronic Engineering Group Bèta, VU University Amsterdam, De Boelelaan 1083, Amsterdam 1081 HV, The Netherlands. ${ }^{4}$ Department of Physics and Astronomy, Space Research Centre, University of Leicester, Leicester LE1 7RH, UK.

Received: 30 January 2015 Accepted: 28 August 2015

Published online: 22 September 2015

\section{References}

1. Fishbaugh KE, Head III JW. Comparison of the North and South Polar Caps of Mars: New Observations from MOLA Data and Discussion of Some Outstanding Questions. Icarus. 2001;154:145-61.

2. Grotzinger J, Beaty D, Dromart G, Gupta S, Harris M, Hurowitz J, et al. Mars Sedimentary geology: Key Concepts and Outstanding Questions. Astrobiology. 2011;11(1):77-87.

3. Levine JS, Garvin JB, Head III JW. Martian Geology Investigations. Planning for the Scientific Exploration of Mars by Humans Cosmology. J Cosmology. 2010;12:3636-46.

4. Hoefen TM, Clark RN, Bandfield JL, Smith MD, Pearl JC, Christensen PR. Discovery of olivine in the Nili Fossae region of Mars. Science. 2003;302:627-30.

5. Mustard JF, Poulet F, Gendrin A, Bibring JP, Langevin Y, Gondet B, et al. Olivine and pyroxene diversity in the crust of Mars. Science. 2005;307:1594-7.

6. Bibring JP, Langevin Y, Mustard JF, Poulet F, Arvidson R, Gendrin A. Global Mineralogical and Aqueous Mars History Derived from OMEGA/Mars Express Data. Science. 2006;312:400-4

7. Andrews-Hanna J C and Lewis K W. Early Mars hydrology: 2. Hydrological evolution in the Noachian and Hesperian epochs. 2011. doi:10.1029/2010JE003709.

8. Pelletier JD, Kolb KJ, McEwen AS, Kirk RL. Recent bright gully deposits on Mars: Wet or dry flow? Geology. 2008;36:211-4.

9. Vítek P, Jehlička J, Edwards H G M, Hutchinson I, Ascaso C, Wierzchos J. Miniaturized Raman instrumentation detects carotenoids in Mars-analogue rocks from the Mojave and Atacama deserts. 2014. doi:10.1098/rsta.2014.0196.

10. Culka A, Košek F, Drahota P, Jehlička J. Use of miniaturized Raman spectrometer for detection of sulfates of different hydration states - Significance for Mars studies. Icarus. 2014;243:440-53.

11. Sharma SK, Misra AK, Lucey PG, Angel SM, McKay CP. Remote pulsed Raman spectroscopy of inorganic and organic materials to a radial distance of 100 meters. Appl Spectrosc. 2006;60:871-6.

12. Dickensheets DL, Wynn-Williams DD, Edwards HGM, Schoen C, Crowder C, Newton EM. A novel miniature confocal microscope/Raman spectrometer system for biomolecular analysis on future Mars missions after Antarctic trials. Journal of Raman Spectroscopy. 2000;31:633-5.

13. Ellery A, Wynn-Williams D. Why Raman spectroscopy on Mars? A case of the right tool for the right job. Astrobiology. 2003;3:565-79.

14. Wang A, Haskin L A, Lane A L, Wdowiak T J, Squyres S W, Wilson R J, Hovland L E, Manatt K S, Raouf N and Smith C D. Development of the Mars microbeam Raman spectrometer (MMRS). 2003. doi:10.1029/2002JE001902.

15. Kolesov BA, Geiger CA. A temperature-dependent single-crystal Raman spectroscopic study of fayalite: evidence for phono-magnetic excitation coupling. Physics and chemistry of minerals. 2004;31:155-61.

16. Knight AK, Scherbarth NL, Cremers DA, Ferris MJ. Characterization of laser induced breakdown spectroscopy (LIBS) for applications to space exploration. Appl Spectrosc. 2000:54:331-40.

17. Mateo-Martí E, Prieto-Ballesteros O, Sobrado JM, Gómez-Elvira J, Martín-Gago JA. A chamber for studying planetary environments and its applications to astrobiology. Sci Technol. 2006;17:2274.

18. de Vera JP, Möhlmann D, Butina F, Lorek A, Wernecke R, Ott S. Survival Potential and Photosynthetic Activity of Lichens Under Mars-Like Conditions: A Laboratory Study. Astrobiology. 2010;10:215-27.

19. Patel MR, Miljkovic K, Ringrose TJ, Leese MR. The Hypervelocity Impact Facility and Environmental Simulation at the Open University. European Planetary Science Congress. 2010;5:655.

20. Sobron P, Wang A. A planetary environment and analysis chamber for combined in situ spectroscopic measurements on selected materials under planetary relevant environments. J. Raman Spectrosc. 2011; doi:10.1002/jrs.3017.

21. Mahaffy PR, Webster CR, Atreya SK, Franz H, Wong M, Conrad PG, et al. Abundance and Isotopic Composition of Gases in the Martian Atmosphere from the Curiosity Rover. Science. 2013;341:263-6.

22. http://www.coolmark.nl/files/productinfo/koudetechniek/danfoss-regelcomponenten/rd6ka502.pdf, 2004. 
23. Welp LR, Keeling RF, Weiss RF, Paplawsky W, Heckman S. Design and performance of a Nafion dryer for continuous operation at $\mathrm{CO}_{2}$ and $\mathrm{CH}_{4}$ air monitoring sites. Atmos Meas Tech Discuss. 2012;5:5449-68.

24. Bazalgette Courreges-Lacoste G, Ahlers B, Rull PF. Combined Raman spectrometer/laser-induced breakdown spectrometer for the next ESA mission to Mars. Spectrochim Acta A Mol Biomol Spectrosc. 2007;68:1023-8.

25. Ahlers B, Hutchinson I, Ingley R, Team, R. L. E. Combined Raman/LIBS spectrometer elegant breadboard-built and tested-and flight model spectrometer unit. Toulouse, France: Proc. 7th Intl. Conf. Space Opt; 2008.

26. Motamedi K. Validating the performance of a Raman laser spectrometer (RLS) instrument under Martian conditions, PhD thesis. Amsterdam, Netherlands: VU University Amsterdam; 2013.

27. Skinner BJ, Porter SC. John Wiley \& Sons. Physical geology. Geological J. 1989;24:160-1.

28. Haymon R M. Growth history of hydrothermal black smoker chimneys, Nature 1983; doi:10.1038/301695a0.

29. Hannington MD, Scott SD. Mineralogy and geochemistry of a hydrothermal silica-sulfide-sulfate spire in the caldera of Axial Seamount, Juan De Fuca Ridge. Can Mineral. 1988;26:603-25.

30. Ingley R, Hutchinson IB, Edwards HGM, Moral AG, Diaz E, Ramos G, et al. ExoMars Raman laser spectrometer breadboard: detector design and performance Proc. SPIE 8152, Instruments, Methods, and Missions for Astrobiology XIV. 2011; doi:10.1117/12.899541.

31. Weber I, Böttger U, Pavlov SG, Jessberger EK, Hübers HW. Mineralogical and Raman spectroscopy studies of natural olivines exposed to different planetary environments. Planet Space Sci. 2014;104:163-72.

32. Jehlicka J, Vítek P and Edwards H G M. Raman spectra of organic acids obtained using a por instrument at $-5^{\circ} \mathrm{C}$ in a mountain area at 2000 m above sea level. JRaman spectrosc. 2009; doi:10.1002/jrs.2450.

33. Weber I, Böttger U, Jessberger EK, Hübers HW, Pavlov SG, Schröder S, et al. Raman spectroscopy of Mars relevant mineral for planetary exploration. The Woodlands, Texas: 43rd Lunar and Planetary Science Conference; 2012.

34. Frech $\mathrm{R}$, Wang EC, Bates JB. The IR and Raman-spectra of $\mathrm{CaCO}_{3}$ (aragonite). Spectrochimica Acta Part A-Molecular and Biomolecular Spectroscopy. 1980;36:915-9.

35. Gunasekaran S, Anbalagan G, Pandi S. Raman and infrared spectra of carbonates of calcite structure. J Raman Spectrosc. 2006;37:892-9.

36. Buzgar N, Buzatu A, Sanislav IV. The Raman study on certain sulfates. Annalele Stiintifice ale Universitatii. 2009:55:5-23.

Submit your manuscript to a SpringerOpen ${ }^{\circ}$ journal and benefit from:

- Convenient online submission

- Rigorous peer review

- Immediate publication on acceptance

Open access: articles freely available online

- High visibility within the field

- Retaining the copyright to your article

Submit your next manuscript at $>$ springeropen.com 